\title{
Rowell Syndrome Presenting as Stevens - Johnson Syndrome / Toxic Epidermal Necrolysis
}

\author{
Barbach Y*, Dah Cherif A, Jroundi C, Chaouche M, Elloudi S, Baybay \\ $\mathrm{H}$ and Zahra Mernissi F \\ Dermatology Department, University Hospital Hassan II, Morocco
}

*Corresponding author: Younes Barbach, Dermatology Department, University Hospital Hassan II, Morocco, Email: dr.younes2011@gmail.com

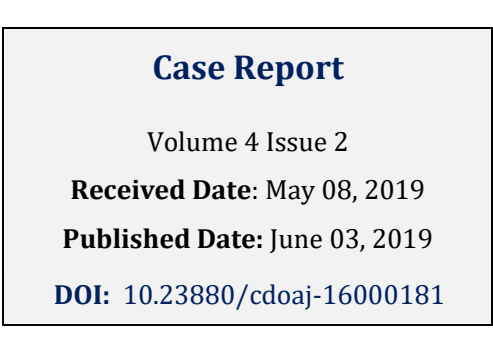

\begin{abstract}
Stevens-Johnson syndrome and toxic epidermal necrolysis are severe forms of cutaneous adverse drug reaction often due to medication. Rarely these cutaneous manifestations can be the first presentation of systemic lupus erythematosus and more rarely in its Rowell Syndrome form. We report a case of a 32-year-old patient who had a clinical presentation of TEN toxidermia and based on several clinical and biological criteria, the diagnosis of Rowell syndrome was established with adequate management of the patient.
\end{abstract}

Keywords: Rowell syndrome; Toxidermia; Lupus

Abbreviations: SJS: Stevens Johnson Syndrome; TEN: Toxic Epidermal Necrolysis; LE: Lupus Erythematosus.

\section{Introduction}

Stevens-Johnson syndrome (SJS) and toxic epidermal necrolysis (TEN) are life-threatening dermatological conditions that are characterized by mucositis, epidermal detachment and erosions. The underlying etiology in SJS and TEN is almost invariably secondary to drugs. Rarely, other causes such as lupus erythematosus (LE), infections and vaccinations have been implicated [1]. Only a few observations of patients with LE that was revealed by a SJS/TEN have been reported, none of which had «Rowell Syndrome".

\section{Case Report}

It was a 32-year-old patient, whose older sister is treated for systemic lupus with renal tropism, with no history of drug intake, consulted for erythematous, bullous itchy and diffuse patches throughout her body evolving for 15 days before her consultation. The dermatological examination revealed the presence of diffuse lesions in cockades and pseudo-cockades predominant on the large folds associated with erythematous erosive patches of the neck, trunk, abdomen and back clear liquid-containing blisters with a positif Nikolsky sign, cutaneous skin surface detached $<5 \%$, the rest of the somatic examination was normal, given the absence of drug intake and the family history of lupus, the diagnosis of Rowell syndrome was mentioned first, a biological assessment was performed confirming the diagnosis with anti-nuclear antibodies positive to $1 / 320$ with speckled pattern, and anti-double-stranded DNA (anti-ds DNA) antibodies and SSA SSB antibodies, the balance of the report did not show systemic signs of lupus, the patient was put under Hydroxychloroquine with all the measures of photo protection with good evolution (Figure 1). 

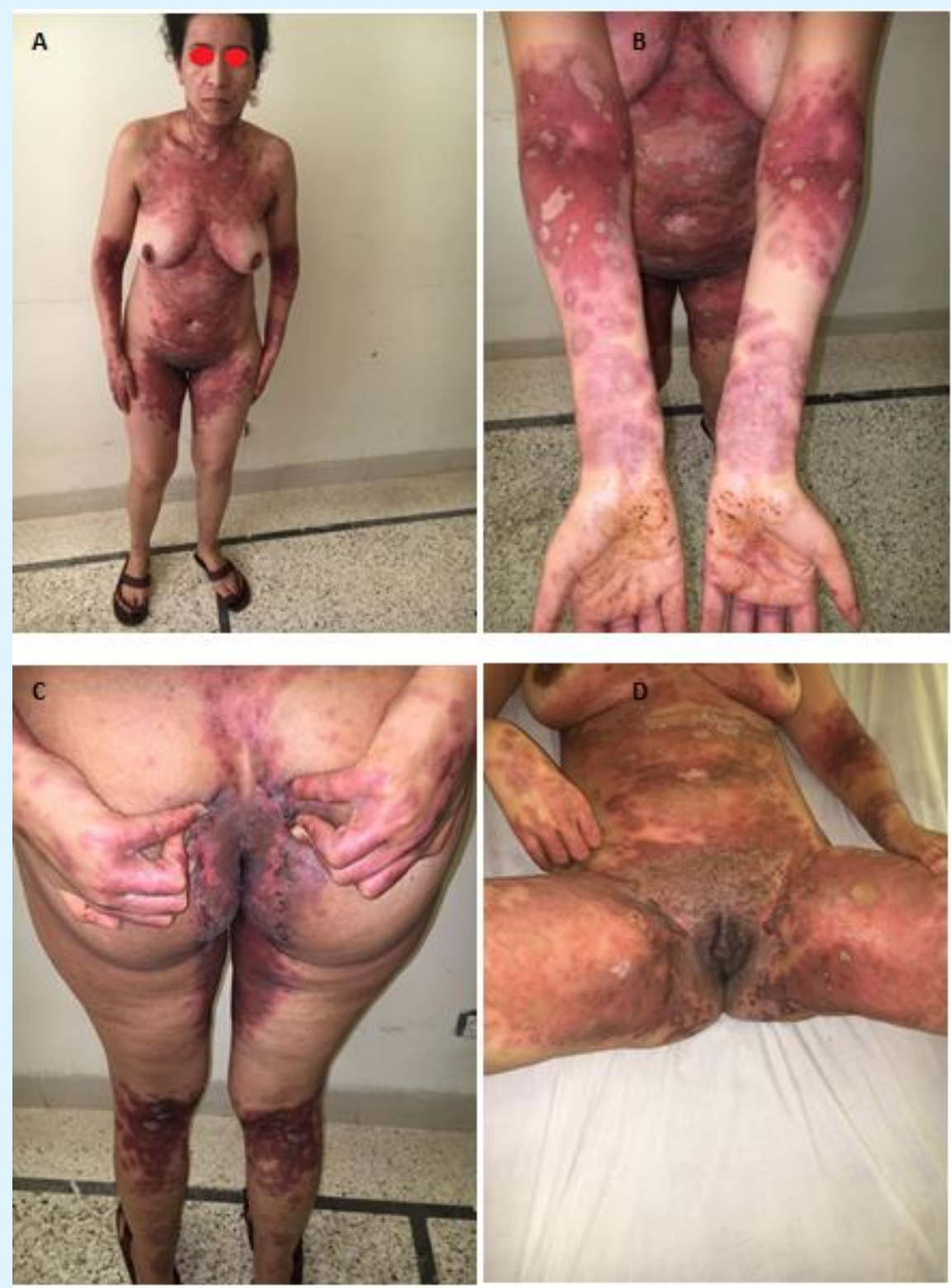

Figure 1: Figures A-B-C-D: Mmultiple diffuse post bullous erosive patches prevailing at the folds and neck and respecting the mucous membranes.

\section{Discussion}

The association of lupus erythematosus and erythema polymorphic is known in the medical literature as Rowell syndrome. This association has been described for the first time by Scholtz in 1922 [2]. In 1963, in a study of 120 patients with discoid lupus erythematosus, Rowell demonstrated in 4 patients erythema multiforme-type lesions associated with discoid lupus erythematosus and specific immunological abnormalities: antinuclear antibodies in speckled fluorescence, anti-Sj-T serum antibodies (which nowadays correspond to anti-La / SSB antibodies) and the presence of a rheumatoid factor. Over the years, Rowell syndrome has been progressively described in association with all types of lupus [3]. Mandelcorn, et al. [4] described for the first time in 2003 two cases of TEN's associated to lupus. Since this first description, some observations have been reported in the literature [5-10]. The topography of cutaneous lesions 
during SJS and TEN's associated with lupus allows a reference to the diagnosis of LE. The involvement of the photo-exposed areas and the realization of the predominant oral mucosa have often been reported in the literature. The suggestive nature of the mucosal lesions highlighted in the first cases of SJS or TEN associated with lupus is not reported in more recent publications [11]. The of distribution lesions suggest the role of ultraviolet rays in triggering the rash.

Medications are the most common cause of SJS and TEN, and the exclusion of causality from the drug is paramount. This involves a detailed history of drugs, focusing on the latency between onset of rash and the relative risk associated with drugs, based on epidemiological surveillance and case monitoring data. In cases of atypical presentation, we will be cautious in assuming drug causality, especially if the latency is greater than 8 weeks or if the drug has already been stopped before the onset of rash or is not a known highrisk drug. Indeed, in large studies, no incriminated drug can be found in approximately 15 to $20 \%$ of patients [1]. In these idiopathic or atypical cases, we recommend screening with ANA to exclude LE, as this may be the initial presentation. Optimal treatment of SJS / TEN lupus remains uncertain. Our patient responded well to hydroxychloroquine treatment with all the measures of photo protection without recurrence after 18 months.

\section{Conclusion}

We report the first case of a patient with an association of Rowell syndrome and SJS / TEN like. Lack of drug intake, family history, clinical features, positivity of NAA, anti-double-stranded DNA (anti-dsDNA) antibodies, SSA SSB antibodies, made it possible to confirm the diagnosis and thus contributed to adequate management.

\section{References}

1. Lee HY, Tey HL, Pang SM, Thirumoorthy T (2011)Systemic lupus erythematosus presenting as Stevens-Johnson syndrome and toxic epidermal necrolysis: a report of three cases. Lupus 20(6): 647652.

2. Scholtz M (1922) Lupus erythematosus acutus disseminatushaemor-rhagicus. Arch Dermatol Syphiligr 6: 466-475.

3. Aydogan K, Karadogan S, Balaban Adim S, Tunali S (2005) Lupus ery-thematosus associated with erythema multiforme: report of two cases and review of the literature. J EurAcad Dermatol Venereol 19(5): 621-267.

4. Mandelcorn R, Shear NH (2003) Lupus-associated toxic epidermal necrolysis: a novel manifestation of lupus?. J Am Acad Dermatol 48(4): 525-529.

5. Paradela S, Martinez-Gomez W, Fernandez-Jorge B, Castiñeiras I, Yebra-Pimentel T, et al. (2007) Toxic epidermal necrolysis-like acute cutaneous lupus erythematosus. Lupus 16(9): 741-475.

6. Cisneros CG, Romiti R, Santi CG, Aoki V, Valente NY, et al. (2010) Toxic epidermal necrolysis-like cutaneous lupus erythematosus: a series of three patients. Acta Derm Venereol 90(2): 175-178.

7. Boontaveeyuwat E, Silpa-archa N, Kulthanan K (2012) Toxic epidermal necrolysis-like acute cutaneous lupus erythematosus (TEN-like ACLE) in SLE patients: a report of two cases. Asian Pac J Allergy Immunol 30(1): 83-87.

8. Ziemer M, Kardaun SH, Liss Y, Mockenhaupt M (2012) Stevens-Johnson syndrome and toxic epidermal necrolysis in patients with lupus erythematosus: a descriptive study of 17 cases from a national registry and review of the literature. Br J Dermatol 166(3): 575-600.

9. Baker MG, Cresce ND, Ameri M, Martin AA, Patterson JW, et al. (2014) Systemic lupus erythematosus presenting as Stevens-Johnson syndrome/toxic epidermal necrolysis. J ClinRheumatol 20(3): 167171.

10. Ranario JS, Smith JL (2014) Bullous lesions in a patient with systemic lupus erythematosus. J ClinAesthet Dermatol 7(9): 44-49.

11. Bellakhal S, Ben Kaab B, Teyeb Z, Souissi A, Derbel F, et al. (2015) Systemic lupus erythematosus presenting as Stevens-Johnson syndrome. Pathol Biol(Paris) 63(4-5): 222-223. 